\title{
Minimization of E2E Delay in Wireless ADHOC Networks based on Residual Energy of Nodes
}

\author{
Prabu V. \\ PG student, Department of ECE, \\ Sri Krishna College of Technology, \\ Coimbatore, TamilNadu, India
}

\author{
Divya K. \\ Assistant Professor, Department of ECE, \\ Sri Krishna College of Technology, \\ Coimbatore, TamilNadu, India
}

\author{
Maheswsr R. \\ Associate Professor, Department of ECE, \\ Sri Krishna College of Technology, \\ Coimbatore, TamilNadu, India
}

\begin{abstract}
In Wireless network, whenever a data is transferred from source to destination, shortest path algorithms are always preferred to minimize delay. As node failures are common in battery operated wireless devices, retransmission has to be carried out when node failure occurs which leads to higher delay and lesser network lifetime. Several algorithms were already proposed to minimize the delay and to maximize the network lifetime. The recently proposed work Reliable Minimum Energy Cost Routing (RMECR) and Reliable Minimum Energy Routing (RMER) considers energyefficiency, reliability and prolonged network lifetime. Here, the energy consumed by each node in the network and the remaining battery energy of the nodes as well as quality of links is considered. This helps in finding an energy-efficient and reliable route that increase the operational lifetime of the network. The generic routing algorithm is implemented to find the residual energy of the nodes and then Dijkstra's algorithm is used to find the shortest path. In this work, Modified Reliable Minimum Energy Cost Routing (MRMECR) is newly proposed where Multipath routing technique is used in which total end-to-end delay is calculated and performance metrics are studied.
\end{abstract}

\section{Keywords}

Hop-by-hop(HBH) and End-2-end(E2E) retransmission, Energyefficiency, Acknowledgement(ACK) packets, wireless Adhoc networks, Multipath routing

\section{INTRODUCTION}

Unlike other wireless networks, Adhoc network is not a centralized network i.e., any node can act as a header node. The primary objective of an adhoc network routing protocol is the correct and efficient route establishment between a pair of nodes so that the message may be delivered reliability and in a timely manner. Because the nodes of an adhoc network are usually small, battery powered devices, energy management is a critical issue for practical deployment of these networks. Energy-efficiency, reliability and prolonging network lifetime are the three main requirements of the wireless adhoc networks and there exists trade-off between latency and energy always ${ }^{[1]}$. Energy-efficiency is the term which gives the details about how powerfully the energy is being used to complete the operation. It is the ratio of output energy to the input energy i.e., remaining energy of the node to the initial energy of that node. Reliability is the term used to analyze whether the information is shared between the medium effectively without having any losses in the information. Since all the devices in the wireless networks are battery operated, the power consumption and power dissipation plays a vital role in extending the lifetime of a network. Several routing algorithms have been proposed to increase energyefficiency, reliability and the lifetime of wireless ad hoc networks. It is broadly classified into three categories:

(1) Algorithms consider reliability of links, ${ }^{[3]}$

(2) Algorithms considered in finding energy-efficient routes, ${ }^{[4],[5],[6],[7]}$

(3) Algorithms that tries to increase the lifetime of the network $^{[8],[9],[10],[12]}$

A new energy-aware routing algorithm called reliable minimum energy cost routing (RMECR) was proposed which finds energy-efficient and reliable routes that increase the operational lifetime of the network. This proposed algorithm works in two types of networks namely hop-by-hop $(\mathrm{HBH})$ retransmission and end-to-end (E2E) retransmission. Based on our detailed and generic analytical model proposed for the design of RMECR in this paper, we devise a state-of-the-art energy-efficient routing algorithm for ad hoc networks called reliable minimum energy routing (RMER). The routes discovered by RMER minimize the consumed energy of the E2E packet traversal in the network. RMER does not consider the remaining battery energy of nodes, and will be used as a benchmark to evaluate energy-efficiency of the RMECR algorithm.

The two existing energy-aware routing algorithms consider single path routing at the time of route discovery. Total End-to-End delay is not taken into account. Hence, a new energy-aware algorithm is proposed namely "Modified Reliable Minimum Energy Cost Routing"(MRMECR) in which the three above mentioned requirements had been fulfilled along with the total end-to-end delay is considered. And finally while comparing the delays of three energy aware algorithms, the delay is minimum for MRMECR algorithm comparing to other two existing algorithms.

\section{METHODOLOGY}

\subsection{ENERGY AWARE RELIABLE ROUTING}

The energy cost of a route is associated to its consistency. The main aim is to find the reliable routes which minimize the energy cost in a system. To do this, reliability and energy cost must 
be considered while selecting the routes between the source and the destination nodes. The reliability and the packet retransmission are inversely proportional where if a routes are less reliable then the packet retransmission increases whereas if the routes are more reliable then the packet retransmission decreases. Hence, a large amount of energy is being consumed for packet retransmissions. In order to compute the energy cost of the routes, two sets of energy aware reliable routing algorithms for $\mathrm{HBH}$ and E2E systems were designed. They are called Reliable Minimum Energy Cost Routing (RMECR) and Reliable Minimum Energy Routing (RMER).

In RMER algorithm, the energy cost of the path is the excepted amount of energy consumed by all the nodes to transfer the packet to the destination. In RMECR algorithm, the energy cost of the path is the expected battery cost of the nodes along the path to transfer a packet from the source to the destination. In MRMECR algorithm, the energy cost of the path is the average residual energy of the path between source and destination. Before proceeding with these algorithms, minimum energy cost path is first defined.

Definition: (Minimum Energy Cost Path).The minimum energy cost path between a source and a destination node is a path which minimizes the expected energy cost for E2E traversal of a packet between the two nodes in a multihop network ${ }^{[2]}$

We design a generic routing algorithm for finding MECP between every two nodes in the network. Since energy cost is the additive metric, it may seem that the Dijkstra's shortest path routing algorithm could be used to find MECP in the both $\mathrm{HBH}$ and E2E system. In the existing work, these two algorithms were implemented using a link state proactive routing protocol such as optimized link state routing (OLSR) protocol. In OLSR protocol, each node shares the information about their network topology with the other nodes sporadically. But in this work, these three algorithms were implemented using a proactive protocol called Adhoc Ondemand Distance Vector routing (AODV). The shortest path between the nodes is calculated using Dijkstra's algorithm. BellmenFord algorithm can also be used to design these three algorithms. Since it has higher computational complexity, Dijkstra's algorithm was implemented. ${ }^{[2]}$

\subsection{HOP-BY-HOP AND END-TO-END RE-TRANSMISSION SYSTEMS}

RMER and RMECR algorithms are implemented in the networks in which either hop-by-hop or end-to-end retransmissions ensure reliability. In general, Wireless networks in the adhoc networks are inclined to transmission errors. Hence retransmission of packets is necessary to ensure reliability. It can be done either using HBH (Hop-by-Hop) or E2E (End-to-End) retransmissions. In the $\mathrm{HBH}$ system, a link level reliability is guaranteed. A lost packet in each hop is retransmitted by the sender. When the receiver receives the packet correctly then it sends back the ACK (acknowledgement) packet to the sender. If the ACK is not received by the sender properly then the sender retransmits the packet. This continues until the sender receives an ACK from the receiver or the maximum number of transmissions attempts is reached. If it is arrived properly then each link will be reliable and the E2E path will also be reliable. In E2E system, the ACKs are generated by the destination node. The retransmission happens only between the source node and the destination nodes. When a destination node receives the packet correctly then it sends a ACK packet to the source node. If the source node doesn't receive any ACKs for the sent packet then it retransmits the packet until it receives the ACK. In both the systems, the retransmissions occur only after the expiration of the timer. In order to prevent unnecessary retransmissions the duration of this timer is assumed to be long.

\subsection{ENERGY AWARE RELIABLE ROUTING ALGORITHMS FOR HBH AND E2E SYSTEMS}

In a HBH system, the energy cost of the path is analysed in four steps:

(1) Examining the expected number of sending data and ACK packets,

(2) Examining the expected energy cost of the link taking into account the energy cost of retransmissions,

(3) Examining the E2E reliability of a path,

(4) Formulating the energy cost of the path taking into account the energy cost of the links and E2E reliability of the paths.

In the E2E system, the energy cost of the path depends on the number of times that the packet and its ACK are transmitted between the source node and the destination node. To determine the energy cost, first E2E reliability of the path for data packets was formulated and E2E ACKs. Then, the expected energy cost is calculated.

2.3.1 EXPECTED NUMBER OF SENDING DATA AND ACK PACKETS. In HBH system, let us assume that a sender node ' $\boldsymbol{p}$ ' is transmitting a packet to the receiver node ' $\boldsymbol{d}$ '. The node ' $\boldsymbol{p}$ ' is supposed to transmit the packet only 'n' times. Since the possibility of packet loss in a wireless links is common, a packet might be retransmitted a random number of times but not greater than n-1. When the node ' $\boldsymbol{d}$ ' receives the packet correctly, then it sends an ACK packet to the sender node. If an ACK reception fails, then an ACK will be retransmitted for the same packet after' $d$ ' receives the packet correctly. Therefore, an ACK will also be transmitted random number of times for the same packet when an ACK is lost but it could not be greater than ' $\boldsymbol{n}$ ' times. When the packet is lost for all ' $\boldsymbol{n}$ ' transmission attempts then no ACK will be transmitted for the data packet. In E2E system, the packet will be transferred from the source node to the destination node. The expected transmission count of packet and ACK will be examined by considering the expected number of times the packet is transmitted from the source node to the destination and the expected number of times ACK is transmitted back from the destination to the source node. Since E2E retransmissions are to guarantee $\mathrm{E} 2 \mathrm{E}$ reliability, hence we assume that the number of E2E retransmissions is large enough.

2.3.2 EXPECTED ENERGY COST OF THE LINK AND PATH. In HBH system, the expected energy cost of the link is examined by considering the total energy consumed by the sender node ' $\boldsymbol{p}$ ' and the total energy considered by the receiver node ' $\boldsymbol{d}$ ' to exchange a packet of length ' $\boldsymbol{L}$ ' bit. In E2E system, the expected energy cost of the path for transferring a data packet from the source node to the destination node is the expected energy cost during a single transmission from the source node to the destination node multiplied by the expected number of times that the source transmits the packet including the first attempt.

2.3.3 RELIABILITY OF THE PATH AND LINK. The reliability of the link is the probability of delivering the packet consecutively to the receiving node within the number of allowed transmissions. In HBH system, the link reliability is related to the Packet Delivery Ratio (PDR) of the link. When the packet is received correctly and its ACK is lost then the packet will be retransmitted after the expiration of timer. If the retransmitted packet is received correctly too, there will be a duplicate packet at the receiver. Duplicate 
packets are usually discarded silently at the MAC layer, but ACKs are sent for them. This, however, affects the energy consumption of the transmitting and the receiving nodes which was considered in computing their energy cost. In E2E system, if the source node does not receive an ACK from the destination then a data packet may be sent again by the source node. It is obvious that there must be reverse path from the destination to the source node to carry the E2E ACK. We assume that this reverse path consist of the same link as that of forward path but in the opposite direction.

\subsection{MODIFIED RELIABLE MINIMUM ENERGY COST ROUTING(MRMECR) ALGORITHM}

Multipath route selection technique is implemented in this algorithm in order to achieve minimum delay in the network without affecting its reliability, energy-efficiency and prolong network lifetime. In the previous work, the researchers had achieved reliability, energy-efficiency and thus improved lifetime of the network. When a node failure occurs in the network, the time it takes to find other energy efficient path will be more in the existing work. Because as soon as a node fails, the source node have to send broadcast message to all nearby nodes expect the malicious or failed node. Then the route selection process has to be implemented again and it takes more time comparing to our method.

In our proposed work, Multipath route selection technique is handled. At the time of route discovery, the source node will find 3 alternate paths to its destination node. Among those 3 paths, the route which has more average remaining energy is selected and the remaining 2 paths will be in sleep mode. When a malicious node is found, the source node will select other energy efficient path immediately from the remaining 2 paths. In this work, the reliability, energy-efficiency and prolong network lifetime is achieved along with minimum end-to-end delay.

2.4.1 Route selection. Using Dijkstra's shortest path algorithm, all the nearby nodes are calculated using minimum distance values. Since a Multipath routing technique is used, it chooses 3 paths based on minimum distance values. The best path among them is selected by calculating the average remaining energy of the path after route discovery messages had been completed.

Let us assume ' $\boldsymbol{i}$ ' be number of nodes where ' $\boldsymbol{i}$ ' varies from 1 to $n$ (last node in the path), E[i] = Remaining energy of the ' ${ }^{\text {th }}{ }^{\text {, }}$, node,

Energy-efficient route,

$$
E-\text { route }=\sum i_{1}^{n} E[i] / n
$$

From the above formula, the average remaining energy of the path is been calculated for each paths separately. The path which has maximum average remaining energy is considered to be the best energy-efficient path.

\section{SIMULATION PARAMETERS}

In this section, the simulation parameters and the value for each parameter was mentioned in the following table. The simulation work was done using Network Simulator 2 tool in the Linux Operating System. This tool consist of two simulation tools namely Network Simulator(ns) and Network Animator(nam).It is composed of two key languages namely $\mathrm{C}++$ and OTcl.

Table 1. Simulation Parameters.

\begin{tabular}{|c|c|}
\hline Parameter & Value \\
\hline Number of Nodes & 200 \\
\hline Size of Network & $1500 \times 1000$ \\
\hline Speed of Node & $10 \mathrm{~m} / \mathrm{sec}$ \\
\hline Transmission range & $250 \mathrm{~m}$ \\
\hline Duration of Simulation & $25 \mathrm{~ms}$ \\
\hline Initial energy & $100 \mathrm{~mJ}$ \\
\hline Transmit power & $0.34 \mathrm{~mW}$ \\
\hline Receive power & $0.16 \mathrm{~mW}$ \\
\hline Traffic type & $\mathrm{CBR}$ \\
\hline Packet size & $512[\mathrm{bytes}]$ \\
\hline
\end{tabular}

\section{SIMULATION RESULTS}

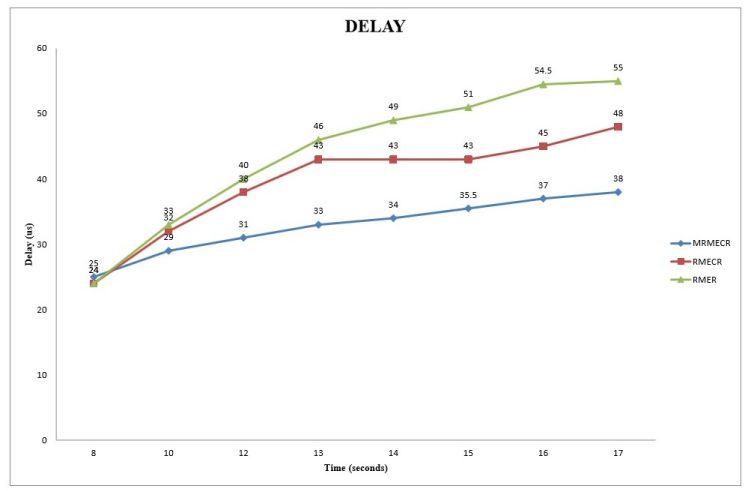

Fig. 1. Average E2E delay vs Time

In figure 1. Our proposed work has minimum E2E delay comparing to the existing work. Our main aim is to reduce total E2E delay by considering the average remaining battery energy of the nodes in the path. The percentage of delay reduced from the existing work is $\mathbf{1 1 . 9 \%}$.

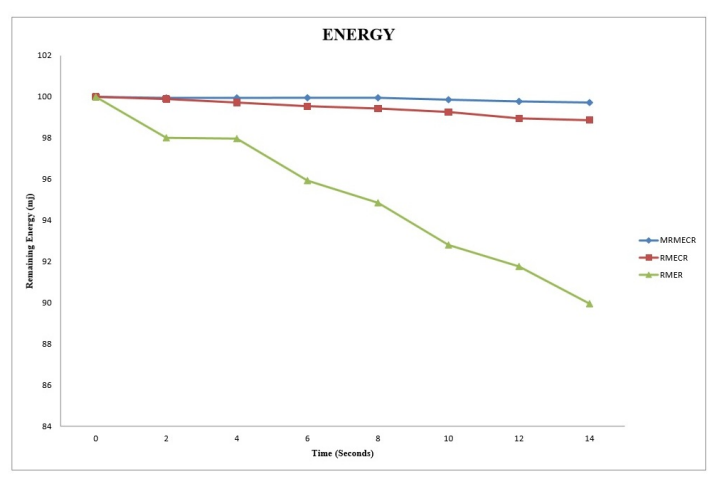

Fig. 2. Remaining Energy vs Time

In Fig. 2, Remaining energy of the path is been found which determines that MRMECR has better path for energy efficiency comparing to other two algorithms.Based on the remaining energy 
of the path, the lifetime of the network is determined. The percentage difference between the existing RMECR and the proposed work is $\mathbf{0 . 4 3 8 \%}$.

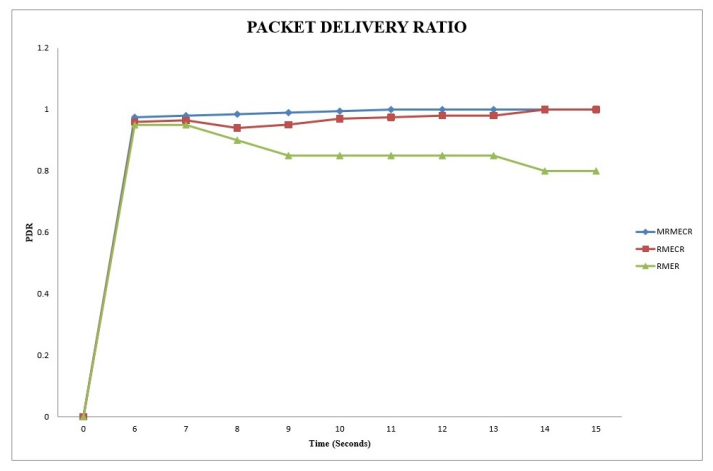

Fig. 3. Packet Delivery Ratio vs Time

In Fig. 3, it shows how successfully the data packets been transferred from the source node to its destination with some minor losses. The comparison graph shows that MRMECR algorithm has better PDR comparing to other two existing algorithms, this is due to the multi path routing technique handled in this algorithm. The best route is choosed by taking the average energy of the nodes in the path makes this algorithm an optimistic one comparing to the other two algorithms and this makes switching of paths without making more delays. The percentage difference for Packet delivery ratio between the existing and the proposed algorithm is $\mathbf{2 . 0 7 \%}$.

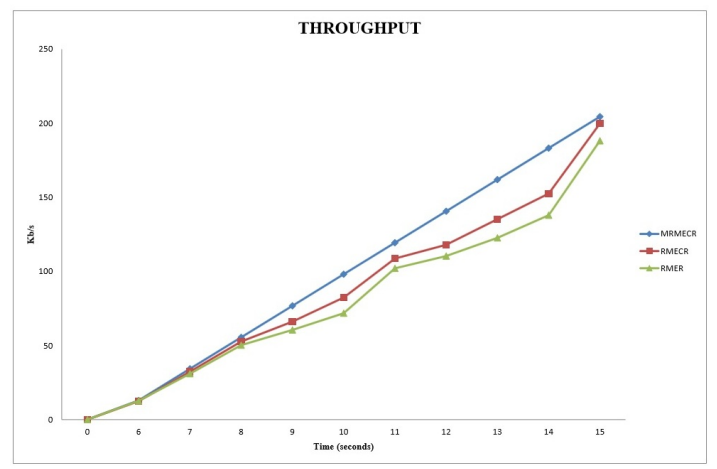

Fig. 4. Throughput vs Time

In Fig. 4, The Throughput is defined as the number of packets been received successfully in the destination for a certain period of time. The throughput is compared, which shows how reliably data be shared in the network. The throughput is better for proposed algorithm compared to our existing algorithms since multi-path routing technique is included in the proposed algorithm. The throughput is achieved to $\mathbf{1 2 . 3 9 \%}$ comparing with the existing work.

In Fig. 5,6,7 and 8, the proposed algorithm is implemented for various network sizes such as for 50,60, 70, 80, 90, 100 nodes respectively.

Thus the simulation results shows the performance comparison of the three algorithms and for the proposed algorithm when a network size is varied.

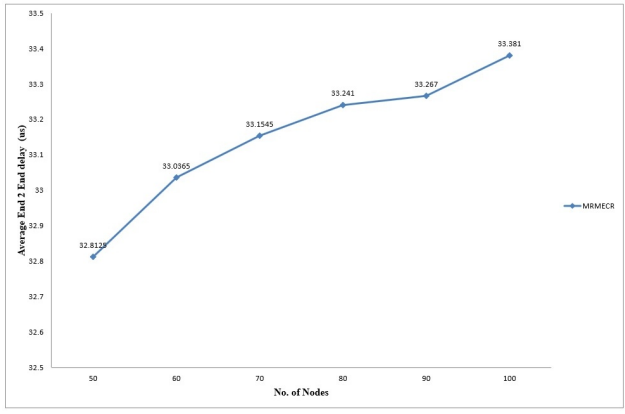

Fig. 5. Average E2E delay vs No. of Nodes

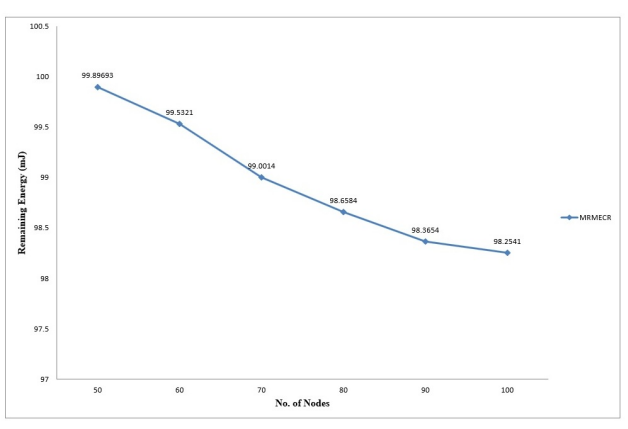

Fig. 6. Remaining Energy vs No. of Nodes

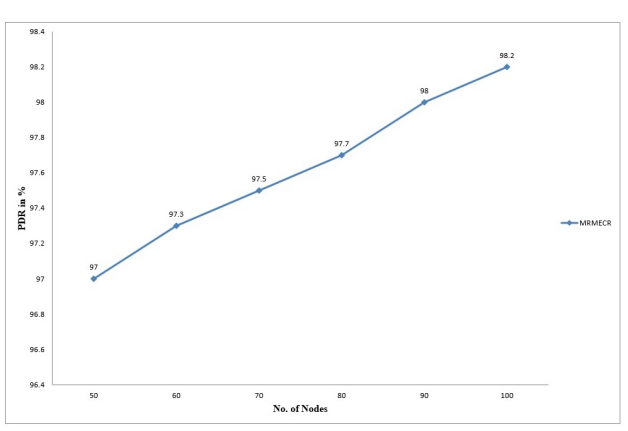

Fig. 7. Packet Delivery Ratio vs No. of Nodes

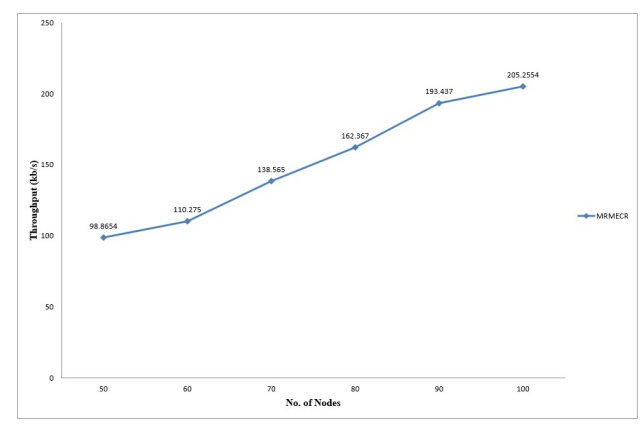

Fig. 8. Throughput vs No. of Nodes 


\section{CONCLUSION}

Thus a new energy aware routing algorithm "Modified Reliable Minimum Energy Cost Routing (MRMECR)" is proposed which reduces the total end-to-end delay in the network along with this, it increases the life-time of the network using energy-efficient and reliable routes. The algorithm considers the average residual energy of the nodes in the path to find the energy efficient route whereas in RMECR algorithm, it considers the remaining battery cost of the node. The simulation result shows that the modified algorithm proves better than the existing algorithms. And in the future work, we planned to implement the Energy harvesting technique to produce more energy in the network thereby improving the life-time of the network.

\section{REFERENCES}

[1] R. Maheswar and R. Jayaparvathy, ?Power Control Algorithm for Wireless Sensor Networks using N-Policy M/M/1 Queueing Model? International Journal on Computer Science and Engineering, Vol 2, No. 7, Dec 2010.

[2] Javad Vazifehdan, R. Venkatesha Prasad, and Ignas Niemegeers, ?Energy-Efficient Reliable Routing Considering Residual Energy in Wireless Ad Hoc Networks?, Proc. IEEE Transactions on Mobile Computing, Vol 13, No 2, Feb 2014.

[3] D.S.J. De Couto, D. Aguayo, J. Bicket, and R. Morris, ?A High-Throughput Path Metric for Multi-Hop Wireless Routing,? Proc. ACM MobiCom, pp. 134-146, 2003.

[4] S. Singh and C. Raghavendra, ?PAMAS?Power Aware MultiAccess Protocol with Signalling for Ad Hoc Networks,? ACM Computer Comm. Rev., vol. 28, pp. 5-26, 1999.

[5] J. Gomez, A.T. Campbell, M. Naghshineh, and C. Bisdikian, ?PARO: Supporting Dynamic Power Controlled Routing in Wireless Ad Hoc Networks,? Wireless Networks, vol. 9, no. 5, pp. 443-460, 2003.

[6] S. Banerjee and A. Misra, ?Minimum Energy Paths for Reliable Communication in Multi-Hop Wireless Networks,? Proc. ACM MobiHoc, pp. 146-156, June 2002.

[7] Q. Dong, S. Banerjee, M. Adler, and A. Misra, ?Minimum Energy Reliable Paths Using Unreliable Wireless Links,? Proc. ACM MobiHoc, pp. 449-459, May 2005.

[8] D.J. Vergados, N.A. Pantazis, and D.D. Vergados, ?EnergyEfficient Route Selection Strategies for Wireless Sensor Networks,? Mobile Networks and Applications, vol. 13, nos. 3-4, pp. 285-296, Aug. 2008.

[9] A. Misra and S. Banerjee, ?MRPC: Maximizing Network Lifetime for Reliable Routing in Wireless Environments,? Proc. IEEE Wireless Comm. and Networking Conf. (WCNC ?02), pp. 800-806, 2002.

[10] S. Singh, M. Woo, and C.S. Raghavendra, ?Power-Aware Routing in Mobile Ad Hoc Networks,? Proc. ACM MobiCom, Oct. 1998.

[11] J. Vazifehdan, R. Prasad, and I. Niemegeers, ?Minimum Battery Cost Reliable Routing in Ad Hoc Wireless Networks,? Proc. Eighth IEEE Consumer Comm. and Networking Conf., Jan. 2011.

[12] C. Toh, ?Maximum Battery Life Routing to Support Ubiquitous Mobile Computing in Wireless Ad Hoc Networks,? IEEE Comm. Magazine, vol. 39, no. 6, pp. 138-147, June 2001.
[13] Pankaj Rohal, Ruchika Dahiya, Prashant Dahiya, and ?Study and Analysis of Throughput, Delay and Packet Delivery Ratio in MANET for Topology Based Routing Protocols (AODV, DSR and DSDV), IJARET Vol 1, Issue 2, March 2013, ISSN: 2320-6802. 